\title{
Application of Scale Relativity (ScR) Theory to the Problem of a Particle in a Finite One-Dimensional Square Well (FODSW) Potential
}

\author{
Saeed Naif Turki Al-Rashid ${ }^{1^{*}}$, Mohammed Abdul-Zahra Habeeb ${ }^{2}$, Khalid Abdulwahab Ahmed ${ }^{3}$ \\ ${ }^{1}$ Physics Department, College of Science, Al-Anbar University, Anbar, Iraq \\ ${ }^{2}$ Physics Department, College of Science, Al-Nahrain University, Baghdad, Iraq \\ ${ }^{3}$ Physics Department, College of Science, Al-Mustansiriyah University, Baghdad, Iraq \\ E-mail:sntr2006@yahoo.com \\ Received April 16, 2011; revised May 22, 2011; accepted June 1, 2011
}

\begin{abstract}
In the present work, and along the lines of Hermann, ScR theory is applied to a finite one-dimensional square well potential problem. The aim is to show that scale relativity theory can reproduce quantum mechanical results without employing the Schrödinger equation. Some mathematical difficulties that arise when obtaining the solution to this problem were overcome by utilizing a novel mathematical connection between ScR theory and the well-known Riccati equation. Computer programs were written using the standard MATLAB 7 code to numerically simulate the behavior of the quantum particle in the above potential utilizing the solutions of the fractal equations of motion obtained from ScR theory. Several attempts were made to fix some of the parameters in the numerical simulations to obtain the best possible results in a practical computer CPU time within limited local computer facilities. Comparison of the present results with the corresponding results obtained from conventional quantum mechanics by solving the Schrödinger equation, shows very good agreement. This agreement was improved further by optimizing the parameters used in the numerical simulations. This represents a new example where scale relativity theory, based on a fractal space-time concept, can accurately reproduce quantum mechanical results without invoking the Schrödinger equation.
\end{abstract}

Keywords: Square Well, ScR Theory, Numerical Simulations, Fractal Space-Time

\section{Introduction}

The extension of the principle of relativity gives new theory of relativity, which is the scale relativity $(\mathrm{ScR})$ theory as introduced by Nottale [1-3] in 1993. This theory states that: "the fundamental laws of nature apply whatever the state of scale of the coordinate system". The state of a reference system is characterized by the resolutions at which this system is observed. It can be defined only in a relative way. The main idea of ScR theory is to give up the arbitrary hypothesis of differentiability of space-time. This theory reformulated quantum mechanics from first principles leading to the covariance and geodesic equations by considering a particle as a geodesic in fractal space-time. ScR theory applies in the three domains of microphysics, cosmology and complex systems [2-9].

As far as quantum mechanics is concerned, Nottale and co-workers were able to apply the theory to solve many problems, especially those related to the conceptual and interpretation aspects. In this connection, we mention the work on the derivation of the postulates of quantum mechanics from the first principles of the ScR theory [10]. In terms of the results of this work, all quantum mechanics and not only the Schrödinger equation, arises as a direct consequence of the fractality of space-time. The extension of the ScR theory to the derivation of the main equations of relativistic quantum mechanics [11] and the relationship between the classical and quantum regimes [12] have been also discussed on the basis of the ScR theory, among other important consequences and implications. With all these far reaching aspects of the theory, direct investigations which would shed light on the basic workings of the ScR theory as formulated by Nottale seem to be warranted.

In this regard, Hermann [13] was the first, and to our 
knowledge, the only researcher, who directly applied the fractal equations of motion obtained from ScR theory in terms of a large number of explicit numerically simulated trajectories for the case of the quantum-mechanical problem of a free particle in an infinite one-dimensional box [14-17]. He constructed a probability density from these trajectories and recovered in this way the solution of the Schrödinger equation without explicitly using it. The results of this work as originally obtained by Hermann [13] are considered as pioneering in this respect since they show the importance of the direct application of ScR theory to quantum systems to reveal how quantum behavior arises from the fractality of space-time. They also demonstrate the validity of this theory and lay the ground for the numerical methods needed in such applications.

It is believed that the results of more applications are important to prove the direct validity of ScR theory in more general cases and not only in a single isolated case as done by Hermann [13]. Besides, such applications are expected to reveal some novel concepts, such as the connection between ScR theory and the Riccati equation [18-21] as revealed in the present work and not observed by Hermann [13] before.

\section{Equation of Motion}

As for a particle in an infinite square well potential, one may start with the complex Newton Equation [10]:

$$
\nabla u=m \frac{\partial}{\mathrm{dt}} \mathbf{V}
$$

where $u$ is a scalar potential and $\mathbf{V}$ is a complex velocity, then separate this equation into real and imaginary parts. Also, for this problem the average classical velocity $\mathbf{v}$ of the particle is expected to be zero [13]. Then, the equations of motion reduce to the forms:

$$
\left.\begin{array}{l}
-D \Delta U-(U . \nabla) U=-\nabla u \\
\frac{\partial}{\mathrm{d} t} U=0
\end{array}\right\}
$$

where $U$ is the imaginary part of complex velocity and $\mathrm{D}$ is the diffusion coefficient. If one takes the $1^{\text {st }}$ of Equations (2) and rewrite it for one-dimension as:

$$
\frac{\partial}{\partial x}\left(\frac{\partial}{\partial x} D U(x)+\frac{1}{2} U^{2}(x)\right)=\frac{1}{m} \frac{\partial}{\partial x} u(x)
$$

then, integrating, one obtains:

$$
D \frac{\partial}{\partial x} U(x)+\frac{1}{2} U^{2}(x)+c_{1}=\frac{1}{m} u(x)
$$

where $c_{1}$ is a constant of integration. According to Hermann's Scale-Relativity method [13], $c_{l}=E / m$. Then,
Equation (4) can be written in the form:

$$
\frac{\mathrm{d}}{\mathrm{d} x} U(x)=-\frac{m}{\hbar} U^{2}(x)+\frac{2}{\hbar}(u(x)-E)
$$

where $D=\frac{\hbar}{2 m}$. Equation (5) has the form of a Riccati

Equation $[18,19]$. To solve this equation, one may transform it into a $2^{\text {nd }}$ order differential equation $[18,19]$ of the form,

$$
r y^{\prime \prime}(x)+r^{2} q(x) y(x)=0
$$

where $[18,19]$,

$$
U(x)=-\frac{1}{r} \frac{y^{\prime}(x)}{y(x)}
$$

and $y(x)$ is an arbitrary function of $x$. From equation (5), it follows that:

$$
r=-\frac{m}{\hbar} ; q(x)=\frac{2}{\hbar}(u(x)-E)
$$

Using Equation (8), Equation (6) becomes,

$$
\frac{\mathrm{d}^{2}}{\mathrm{~d} x^{2}} y(x)-\frac{2 m}{\hbar^{2}}(u(x)-E) y(x)=0
$$

Depending on the values of $E$, there are two general classes for the solution of Equation (9) $[14,22,23]$ which are:

- Bound state solution if $E<u_{0}$; the particle is confined to the region of potential well.

- Free particle state solution if $E>u_{0}$; the particle is free to reach $x= \pm \infty$.

In the present problem, the case $E<u_{0}$ is assumed. There are three regions of potential in the problem of a finite square well that are [14,22]:

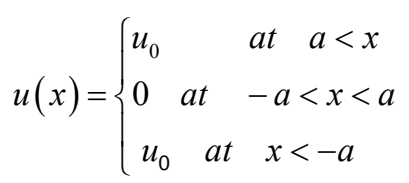

Then, the general solutions of Equation (9) are given by $[14-17,23]$ :

$$
\left.\begin{array}{l}
y_{1}(x)=G e^{K x}+G^{\prime} e^{-K x} \quad \text { for } \quad x<-a \\
y_{2}(x)=A \cos \kappa x+B \sin \kappa x \quad \text { for } \quad-a<x<a \\
y_{3}(x)=H^{\prime} e^{K x}+H e^{-K x} \quad \text { for } \quad a<x
\end{array}\right\}
$$

where $G, G^{\prime}, A, B, H^{\prime}$ and $\mathrm{H}$ are arbitrary constants, $\kappa=\sqrt{2 m E / \hbar^{2}}$ and $K=\sqrt{2 m\left(u_{0}-E\right) / \hbar^{2}}$. Applying the boundary conditions at $x \rightarrow \pm \infty$, this leads to $y(x)$ $\rightarrow 0$. Then, one can rewrite Equations (11) as: 


$$
\left.\begin{array}{l}
y_{1}(x)=G e^{K x} \\
y_{2}(x)=A \cos \kappa x+B \sin \kappa x \\
y_{3}(x)=H e^{-K x}
\end{array}\right\}
$$

The next step is to apply the matching conditions at the boundaries between regions, which require that both function and its derivative be continuous. In this way, one gets a set of four homogeneous linear equations with four unknowns [19]:

(1)

$$
\left.\begin{array}{l}
G e^{-K x}=A \cos \kappa a-B \sin \kappa a \\
K G e^{-K a}=\kappa A \sin \kappa a+\kappa B \cos \kappa a
\end{array}\right\} \text { for } \quad x=-a
$$

(2)

$$
\left.\begin{array}{l}
A \cos \kappa a+B \sin \kappa a=H e^{-K a} \\
-\kappa A \sin \kappa a+\kappa B \cos \kappa a=-K H e^{-K a}
\end{array}\right\} \quad \text { for } \quad x=a
$$

These equations can be rewritten in matrix form as:

$$
\left(\begin{array}{cccc}
\cos \kappa a & -\sin \kappa a & -e^{-K a} & 0 \\
\kappa \sin \kappa a & \cos \kappa a & -K e^{-K a} & 0 \\
\cos \kappa a & \sin \kappa a & 0 & e^{-K a} \\
-\kappa \cos \kappa a & \kappa \cos \kappa a & 0 & K e^{-K a}
\end{array}\right)\left(\begin{array}{l}
A \\
B \\
G \\
H
\end{array}\right)=\boldsymbol{M}\left(\begin{array}{l}
A \\
B \\
G \\
H
\end{array}\right)=0
$$

where the matrix $\boldsymbol{M}$ is given by:

$$
\boldsymbol{M}=\left(\begin{array}{cccc}
\cos \kappa a & -\sin \kappa a & -e^{-K a} & 0 \\
\kappa \sin \kappa a & \kappa \cos \kappa a & -K e^{-K a} & 0 \\
\cos \kappa a & \kappa \sin \kappa a & 0 & e^{-K a} \\
-\kappa \cos \kappa a & \kappa \cos \kappa a & 0 & K e^{-K a}
\end{array}\right)
$$

The trivial solution of Equation (15) is $A=0, B=0, G$ $=0$ and $H=0$ [23]. While, for a non-trivial solution to exist, the condition [23]:

$$
\operatorname{det} \boldsymbol{M}=0
$$

must be satisfied. To simplify, one eliminates the coefficients $G$ and $H$. Then, Equation (15) becomes the $2 \times 2$ matrix equation [23]:

$$
\left(\begin{array}{cc}
\kappa \tan \kappa a-K & K \tan \kappa a+\kappa \\
-\kappa \tan \kappa a+K & K \tan \kappa a+\kappa
\end{array}\right)\left(\begin{array}{l}
A \\
B
\end{array}\right)=M^{\prime}\left(\begin{array}{l}
A \\
B
\end{array}\right)=0
$$

For this equation to have a non-trivial solution, the determinant of the coefficients must be equal to zero, or:

$$
2(\kappa \tan \kappa a-K)(K \tan \kappa a+\kappa)=0
$$

Then, there are two solutions which are [23]:

(a) $\kappa \tan \kappa a=K$, this means $B=0$ and

$$
y_{2}(x)=A \cos \kappa x
$$

(b) $K \tan \kappa a=\kappa$, this means $A=0$ and

$$
y_{2}(x)=B \sin \kappa x
$$

Equation (20) corresponds to even parity solutions while Equation (21) corresponds to odd parity solutions. These equations can be simplified by introducing the new dimensionless variables:

$$
x=\kappa a \text { and } \eta=K a
$$

From the definition of $\kappa$ and $K$, one can write:

$$
\kappa^{2}+K^{2}=\frac{2 m}{\hbar^{2}} u_{0}
$$

Using Equation (22), one can rewrite Equations (20), (21) and (23) in the forms:

$$
\begin{gathered}
\eta=x \tan x \\
\eta=-x \cot x \\
\eta^{2}+x^{2}=\frac{2 m}{\hbar^{2}} u_{\circ} a^{2}=\alpha^{2}
\end{gathered}
$$

where the dimensionless parameter $\alpha$ measures the volume of the potential $u_{0} a^{2}$ in unit of $\hbar^{2} / 2 \mathrm{~m}$.

To determine the values of $\kappa$ and $K$ in Equations (24) and (25), one may solve these equations graphically together with Equation (26) [14-17,22,23].

Figures (1) and (2) give the intercepts for the even parity solution (Equation (24)) and the odd parity solution (Equation (25)) for two sets of values of the potential volume parameter $(\alpha=1$ and 4$)$ and $(\alpha=2$ and 6 ) for the even and odd parity solutions respectively. In these figures, $\kappa$ is drawn horizontally and $\eta$ vertically. The dashed curve is that of $x \tan x$ (for even parity) or $x \cot x$ (for odd parity). The continuous curve is that of $\eta^{2}+x^{2}=\alpha^{2}$

The values of $\kappa$ and $K(x$ and $\eta)$ corresponding to the solutions of Equations (24), (25) and (26) can be determined from Figures (1) and (2). Then, one can calculate the state energy and the function $y(x)$ for different values of $\alpha$ in the following way:

(1) For $\alpha=1$ (equivalent to $\mathrm{n}=1$ ), $x \simeq 0.7391$ and $\eta=$ 0.673 . This is called the ground state energy, which is:

$$
E_{g s} \approx(0.7391)^{2} \frac{\hbar^{2}}{2 m a^{2}}=0.273 \frac{\hbar^{2}}{m a^{2}}
$$




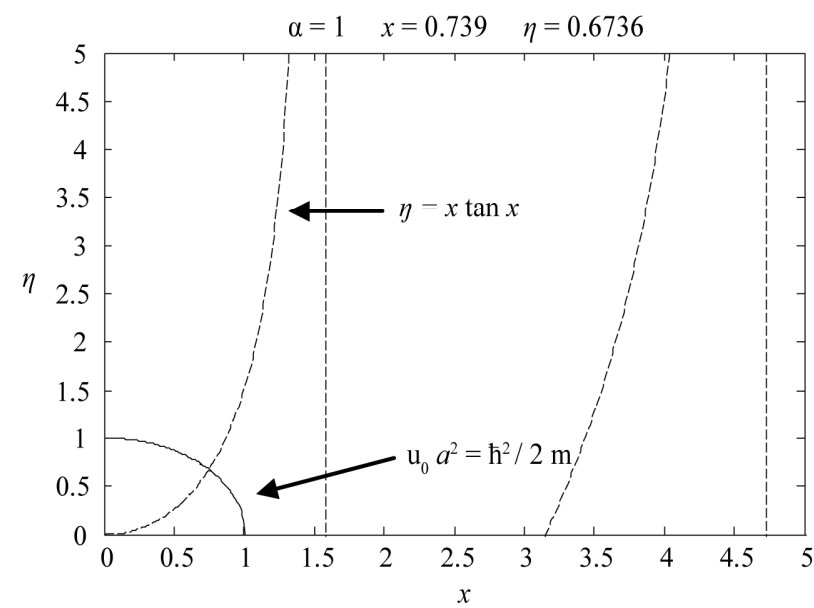

(a)

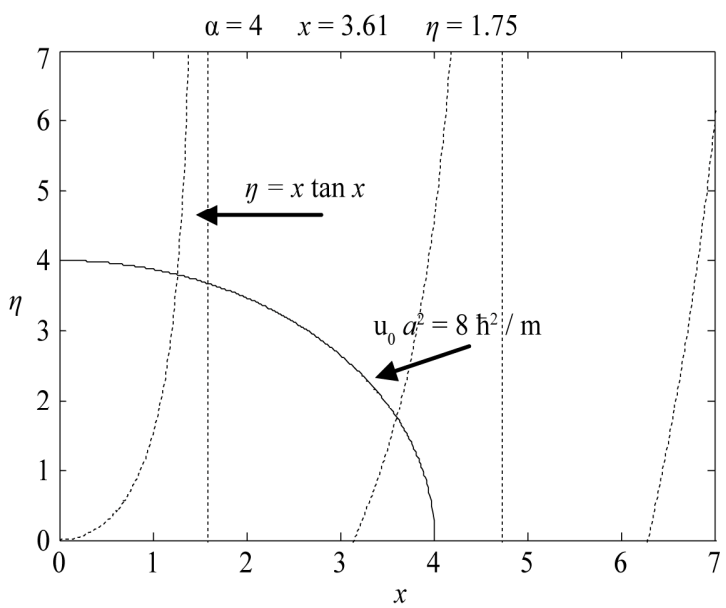

(b)

Figure 1. Graphical solution of Equation (24) (even parity solution), for (a) $\alpha=1$ and (b) $\alpha=4$.

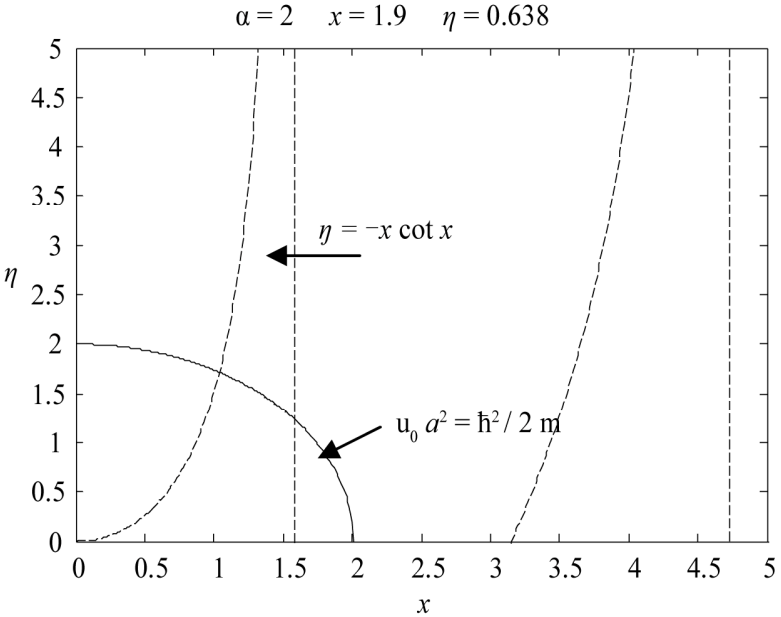

(a)

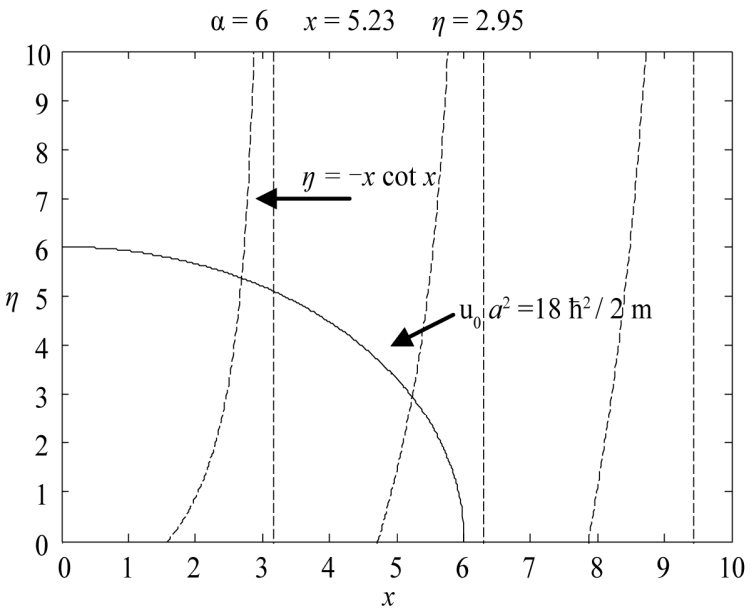

(b)

Figure 2. Graphical solution of Equation (25) (odd parity solution), for (a) $\alpha=2$ and (b) $\alpha=6$.

and Equation (12) can be re-written for even parity solutions as:

$$
y(x)=\left\{\begin{array}{l}
G \exp \left(\frac{0.673}{a} x\right) \quad \text { for } \quad x<-a \\
A \cos \left(\frac{0.7391}{a} x\right) \text { for } \quad-a<x<a \\
H \exp \left(-\frac{0.673}{a} x\right) \text { for } x>a
\end{array}\right.
$$

According to Equation (7), the function $U(x)$ can be defined, using Equation (28), as:

$$
U(x)=\frac{\hbar}{m a}\left\{\begin{array}{l}
0.673 \text { for } x<-a \\
-0.739 \tan \left(\frac{0.739}{a} x\right) \text { for } \quad-a<x<a \\
-0.673 \text { for } x>a
\end{array}\right.
$$

As in Hermann [13], $U(x)$ is treated as a difference of velocities, i.e., it is a kind of acceleration. Thus, the equation of position coordinate (2) has the following form, which is a stochastic process [13]:

$\mathrm{d} x(t)=$

$$
\frac{\hbar}{m a}\left\{\begin{array}{l}
0.673 . \mathrm{d} t+\mathrm{d} \xi_{+}(t) \quad \text { for } \quad x<-a \\
-0.739 \tan \left(\frac{0.739}{a} x\right) \mathrm{d} t+\mathrm{d} \xi_{+}(t) \quad \text { for } \quad-a<x<a \\
-0.673 . \mathrm{d} t+\mathrm{d} \xi_{+}(t) \text { for } x>a
\end{array}\right.
$$

(2) For $\alpha=2$ (equivalent to $n=2$ ), $x \simeq 1.9$ and $\eta=$ 0.638 , the energy is:

$$
E \approx(1.9)^{2} \frac{\hbar^{2}}{2 m a^{2}}=1.805 \frac{\hbar^{2}}{m a^{2}}
$$

and Equation (12) can be re-written for odd parity solu- 
tions as:

$$
y(x)=\left\{\begin{array}{l}
G \exp \left(\frac{0.638}{a} x\right) \quad \text { for } \quad x<-a \\
B \sin \left(\frac{1.9}{a} x\right) \text { for } \quad-a<x<a \\
H \exp \left(-\frac{0.638}{a} x\right) \text { for } x>a
\end{array}\right.
$$

$$
\mathrm{d} x(t)=
$$$$
\frac{\hbar}{m a}\left\{\begin{array}{l}
1.75 \mathrm{~d} t+\mathrm{d} \xi_{+}(t) \quad \text { for } \quad x<-a \\
-3.61 \tan \left(\frac{3.61}{a} x\right) \mathrm{d} t+\mathrm{d} \xi_{+}(t) \quad \text { for } \quad-a<x<a \\
-1.75 \mathrm{~d} t+\mathrm{d} \xi_{+}(t) \text { for } x>a
\end{array}\right.
$$

Also,

$$
U(x)=\frac{\hbar}{m a}\left\{\begin{array}{l}
0.638 \text { for } x<-a \\
1.9 \cot \left(\frac{1.9}{a} x\right) \text { for }-a<x<a \\
-0.638 \text { for } x>a
\end{array}\right.
$$

and,

$$
\begin{aligned}
& \mathrm{d} x(t)= \\
& \frac{\hbar}{m a}\left\{\begin{array}{l}
0.638 . \mathrm{d} t+\mathrm{d} \xi_{+}(t) \quad \text { for } \quad x<-a \\
1.9 \cot \left(\frac{1.9}{a} x\right) \mathrm{d} t+\mathrm{d} \xi_{+}(t) \text { for } \quad-a<x<a \\
-0.638 . \mathrm{d} t+\mathrm{d} \xi_{+}(t) \text { for } \quad x>a
\end{array}\right.
\end{aligned}
$$

(3) For $\alpha=4$ (equivalent to $n=3$ ), $x=3.61$ and $\eta=$ 1.75 , the energy is:

$$
E \approx 6.51 \frac{\hbar^{2}}{m a^{2}}
$$

and Equation (12) for even parity solutions becomes:

$$
y(x)=\left\{\begin{array}{l}
G \exp \left(\frac{1.75}{a} x\right) \text { for } \quad x<-a \\
A \cos \left(\frac{3.61}{a} x\right) \text { for } \quad-a<x<a \\
H \exp \left(-\frac{1.75}{a} x\right) \text { for } x>a
\end{array}\right.
$$

Again,

$$
U(x)=\frac{\hbar}{m a}\left\{\begin{array}{l}
1.75 \text { for } x<-a \\
-3.61 \tan \left(\frac{3.61}{a} x\right) \quad \text { for } \quad-a<x<a \\
-1.75 \text { for } x>a
\end{array}\right.
$$

and,
(4) For $\alpha=6$ (equivalent to $n=4$ ), $x=5.23$ and $\eta=$ 2.95 , the energy is:

$$
E \approx 13.6 \frac{\hbar^{2}}{m a^{2}}
$$

and Equation (12) for odd parity solutions becomes:

$$
y(x)=\left\{\begin{array}{l}
G \exp \left(\frac{2.95}{a} x\right) \quad \text { for } \quad x<-a \\
B \sin \left(\frac{5.23}{a} x\right) \quad \text { for } \quad-a<x<a \\
H \exp \left(-\frac{2.95}{a} x\right) \text { for } x>a
\end{array}\right.
$$

Also,

$$
U(x)=\frac{\hbar}{m a}\left\{\begin{array}{l}
2.95 \text { for } x<-a \\
5.23 \cot \left(\frac{5.23}{a} x\right) \quad \text { for } \quad-a<x<a \\
-2.95 \text { for } x>a
\end{array}\right.
$$

and,

$$
\begin{aligned}
& \mathrm{d} x(t)= \\
& \frac{\hbar}{m a}\left\{\begin{array}{l}
2.95 \mathrm{~d} t+\mathrm{d} \xi_{+}(t) \text { for } x<-a \\
5.23 \cot \left(\frac{5.23}{a} x\right) \mathrm{d} t+\mathrm{d} \xi_{+}(t) \text { for }-a<x<a \\
-2.95 \mathrm{~d} t+\mathrm{d} \xi_{+}(t) \text { for } x>a
\end{array}\right.
\end{aligned}
$$

where $\mathrm{d} \xi_{+}(t)$ is a random variable of a Gaussian distribution with width $\sqrt{2 D \mathrm{~d} t} \quad$ [13].

\section{Numerical Simulations}

To simplify Equations (30), (34), (38) and (42), one can take $2 D \mathrm{dt}=1$ [13], then, these equations become:

(1) $\alpha=1$ 


$$
\begin{aligned}
& \mathrm{d} x(t)= \\
& \frac{1}{a}\left\{\begin{array}{l}
0.673+N(0,1) \text { for } x<-a \\
-0.739 \tan \left(\frac{0.739}{a} x\right)+N(0,1) \text { for }-a<x<a \\
-0.673+N(0,1) \text { for } x>a
\end{array}\right.
\end{aligned}
$$

(2) $\alpha=2$

$$
\begin{aligned}
& \mathrm{d} x(t)= \\
& \frac{1}{a}\left\{\begin{array}{l}
0.638+N(0,1) \text { for } x<-a \\
1.9 \cot \left(\frac{1.9}{a} x\right)+N(0,1) \text { for }-a<x<a \\
-0.638+N(0,1) \text { for } x>a
\end{array}\right.
\end{aligned}
$$

(3) $\alpha=4$

$$
\begin{aligned}
& \mathrm{d} x(t)= \\
& \frac{1}{a}\left\{\begin{array}{l}
1.75+N(0,1) \text { for } x<-a \\
-3.61 \tan \left(\frac{3.61}{a} x\right)+N(0,1) \text { for }-a<x<a \\
-1.75+N(0,1) \text { for } x>a
\end{array}\right.
\end{aligned}
$$

(4) $\alpha=6$

$$
\begin{aligned}
& \mathrm{d} x(t)= \\
& \frac{1}{a}\left\{\begin{array}{l}
2.95+N(0,1) \text { for } x<-a \\
5.23 \cot \left(\frac{5.23}{a} x\right)+N(0,1) \text { for }-a<x<a \\
-2.95+N(0,1) \text { for } x>a
\end{array}\right.
\end{aligned}
$$

where $N(0,1)$ is a normalized random variable [10].

A computer program was written (see Appendix A), following Hermann's procedure [13], to make numerical simulations for the FODSW problem. Numerical simulations are performed using Equations (43), (44), (45) and (46) which represent trajectory equations of the particle for different value of $\alpha$. The output of these simulations gives the probability density $f(x)$ of the particle in a finite square well potential. To construct it, one may divide the region into 1800 pieces (boxes), which gives the best results. This choice comes after many numerical tests. Here, one chooses the time step equal to $5 \times 10^{8}$ which gives best results also after many numerical tests. The $x$ position in the region will be drawn horizontally and the number of occurrences vertically. So, a point of the curves to be drawn has to be understood as $(x, y) ; x$ is the number of boxes and $y$ is the number of time steps for which the particle was in box $x$. The continuous curves indicate the results of the present simulations and the dashed curves the results of conventional quantum mechanics, with the same normalization as the numerical results. The results are always normalized by multiplying the number of occurrences in each box by the total number of boxes which is $a$.

The probability density $P(x)$ of conventional quantum mechanics, which will be compared with the present results, is given by:

(1) For even parity solutions:

$$
\begin{aligned}
& P(x)= \\
& \left\{\begin{array}{l}
\tilde{N}_{1}^{2} \exp \left(2 \eta \frac{x}{a}\right) \text { for } x<-a \\
\tilde{N}_{2}^{2} \cos ^{2}\left(x \frac{x}{a}\right) \text { for }-a<x<a \\
\tilde{N}_{3}^{2} \exp \left(-2 \eta \frac{x}{a}\right) \text { for } x>a
\end{array}\right.
\end{aligned}
$$

(2) For odd parity solutions:

$$
\begin{aligned}
& P(x)= \\
& \left\{\begin{array}{l}
\tilde{N}_{4}^{2} \exp \left(2 \eta \frac{x}{a}\right) \quad \text { for } \quad x<-a \\
\tilde{N}_{5}^{2} \sin ^{2}\left(x \frac{x}{a}\right) \text { for } \quad-a<x<a \\
\tilde{N}_{6}^{2} \exp \left(-2 \eta \frac{x}{a}\right) \text { for } x>a
\end{array}\right.
\end{aligned}
$$

where $\tilde{N}_{1}, \ldots, \tilde{N}_{6}$ are normalization constants [14-18].

For $\alpha=1,2,4$ and 6 this probability density is given by [23]:

(1) $\alpha=1$

$$
P(x)=\frac{1}{a}\left\{\begin{array}{l}
0.844 \exp \left(1.3 \frac{x}{a}\right) \quad \text { for } \quad x<-a \\
0.4019 \cos ^{2}\left(0.739 \frac{x}{a}\right) \quad \text { for }-a<x<a \\
0.844 \exp \left(-1.3 \frac{x}{a}\right) \text { for } x>a
\end{array}\right.
$$

(2) $\alpha=2$

$$
P(x)=\frac{1}{a}\left\{\begin{array}{l}
1.4304 \exp \left(1.27 \frac{x}{a}\right) \quad \text { for } \quad x<-a \\
0.445 \sin ^{2}\left(1.9 \frac{x}{a}\right) \quad \text { for } \quad-a<x<a \\
1.4304 \exp \left(-1.27 \frac{x}{a}\right) \quad \text { for } x>a
\end{array}\right.
$$


(3) $\alpha=4$

$$
P(x)=\frac{1}{a}\left\{\begin{array}{lll}
16.828 \exp \left(3.5 \frac{x}{a}\right) & \text { for } & x<-a \\
0.638 \cos ^{2}\left(3.61 \frac{x}{a}\right) & \text { for } & -a<x<a \\
16.828 \exp \left(-3.5 \frac{x}{a}\right) & \text { for } & x>a
\end{array}\right.
$$

(4) $\alpha=6$

$$
P(x)=\frac{1}{a}\left\{\begin{array}{lll}
227.37 \exp \left(5.9 \frac{x}{a}\right) & \text { for } & x<-a \\
0.8244 \sin ^{2}\left(5.23 \frac{x}{a}\right) & \text { for } & -a<x<a \\
227.37 \exp \left(-5.9 \frac{x}{a}\right) & \text { for } & x>a
\end{array}\right.
$$

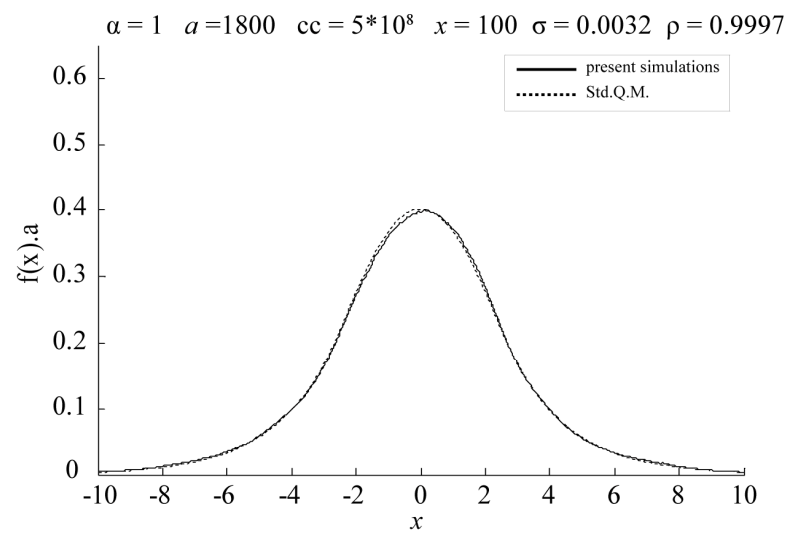

(a)
The comparison between the present results and the results of conventional quantum mechanics is further facilitated by calculating the standard deviation $\sigma$ and correlation coefficient $\rho$, which are given by [13]:

$$
\sigma=\sqrt{\frac{\sum_{i=1}^{N}(P(i)-f(i))^{2}}{N}}
$$

and

$$
\rho=\frac{\sum_{i=1}^{N}(P(i)-<P>)(f(\mathrm{i})-<f>)}{\sum_{\mathrm{i}=1}^{N}(P(i)-<P>)^{2} \sum^{N}{ }_{i=1}(f(\mathrm{i})-<f>)^{2}}
$$

where $N$ is the number of pieces, $P(i) \equiv P(x)$ and $f(i) \equiv$ $f(x)$.

Figures (3) and (4) show a first attempt of modeling for $\alpha=1$ and 4 (even parity solutions) and for $\alpha=2$ and 6

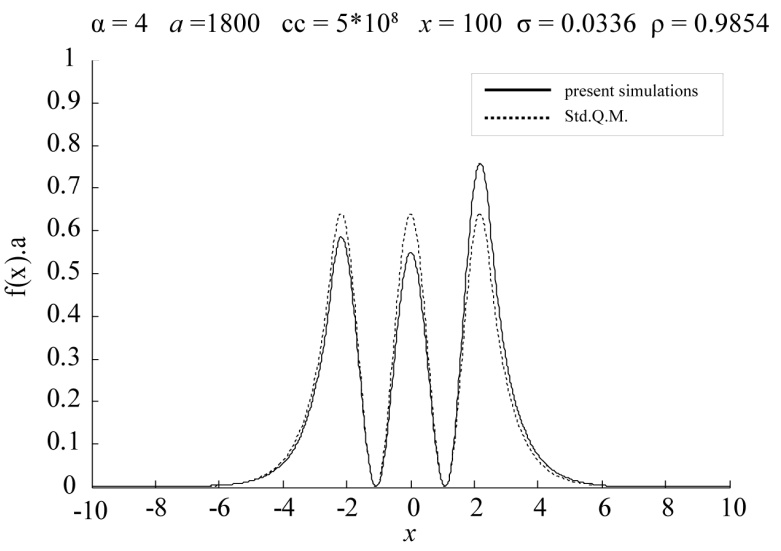

(b)

Figure 3. Probability density for even parity solutions corresponding to a particle in a FODSW potential (a) $\alpha=1$ and (b) $\alpha=$ 4 without thermalization process.

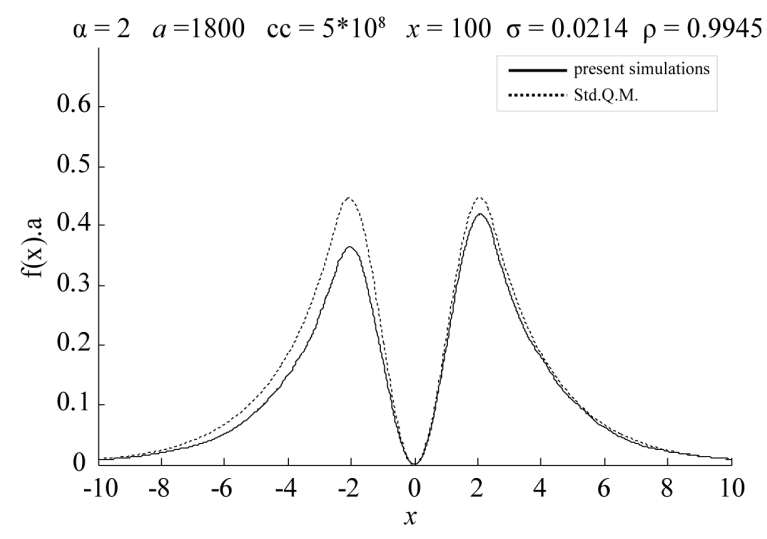

(a)

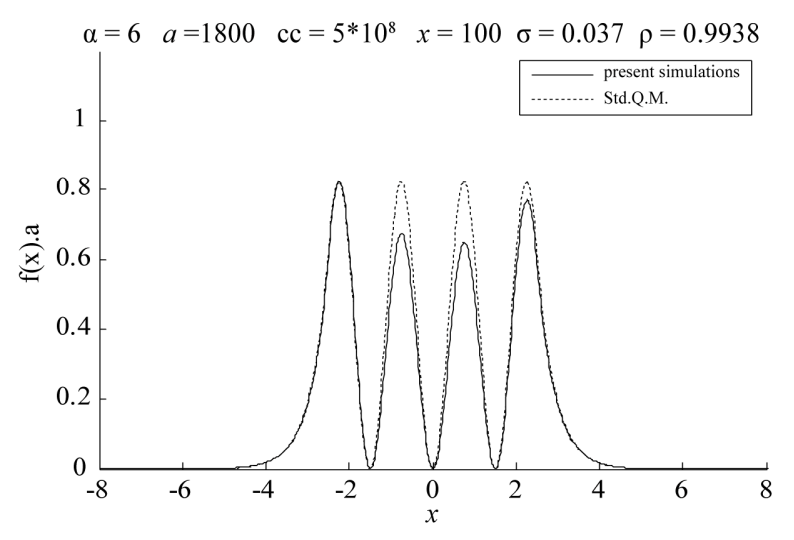

(b)

Figure 4. Probability density for odd parity solutions corresponding to a particle in a FODSW potential (a) $\alpha=2$ and (b) $\alpha=$ 6, without thermalization process. 


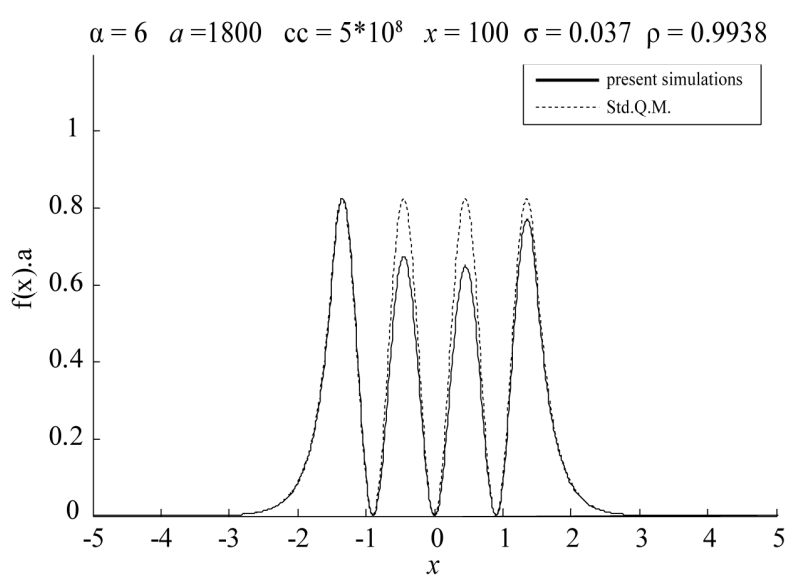

Figure 5. Probability density for $\alpha=6$ (odd parity solution) corresponding to a particle in a FODSW potential after increasing the number of boxes.

(odd parity solutions) respectively. The numerical simulations start with arbitrary point which is $x=100$ (corresponding to box No. 100). In these figures, there is a clear difference between the present results and the results of quantum mechanics, that is measured by $\sigma$ and $\rho$.

Hermann [13] indicated in his work that the simulations were restarted after $10^{5}$ steps, or more, with a new starting position. Then, better thermalization of the system is obtained and convergence is increased. Tests in the present work indicated that the thermalization process as used by Hermann [13] cannot be applied here without fixing additional parameters. This required very long computer time and, therefore, was not adopted in the present work. However, these tests also indicated that the present results can be improved by increasing the number of divisions of $a$ (i.e., number of boxes). Figure (5) shows the results obtained for $\alpha=6$ after increasing the number of boxes from 1800 to 2200 .

\section{Conclusions}

It can be seen from the present work that the behavior of a quantum particle in an infinite one-dimensional square well potential can be obtained without explicitly writing the Schrödinger equation or using any conventional quantum axiom. This leads one to conclude from the present work that $\mathrm{ScR}$ is a well-founded theory for deriving quantum mechanics from the concept of fractal space-time.

Even though many of the aspects of Hermann's work were used in the present work as they are, the application of his approach to the present quantum mechanical problem was not a direct one. Successful applications were not achievable without, among other things, a new adjustment for the time step dt after some deeper understanding of the underlying particle motion in the present problem. It is expected that this understanding is neces- sary when attempts are made to solve other quantum mechanical problems along the lines of Hermann's work [13] and the present work. It is also concluded from the attempts made in the present work to improve the results of numerical simulation by parameter optimization, that such attempts are successful in achieving some improvement, and that further improvement is possible, but requires more computer time.

\section{Acknowledgments}

We would like to deeply thank Prof. Dr. L. Nottale (Director of Research, CNRS, Paris, France) for clarifying some points regarding his theory of scale relativity and for supplying some literature and Dr. R. Hermann (Dept. of Physics, Univ. de Liege, Belgium) for his suggestions concerning further applications of the theory of scale relativity.

\section{References}

[1] L. Nottale, "The Theory of Scale Relativity," International Journal of Modern Physics A, Vol. 7, No. 20, 1992, pp. 4899-4936. doi:10.1142/S0217751X92002222

[2] L. Nottale, "Fractal Space-Time and Microphysics: Towards a Theory of Scale Relativity," World Scientific, Singapore, 1998.

[3] L. Nottale, "The Scale Relativity Program," Chaos, Solitons and Fractals, Vol. 10, No. 2-3, 1994, pp. 459-468. doi:10.1016/S0960-0779(98)00195-7

[4] L. Nottale, "Scale Relativity and Fractal Space-Time: Application to Quantum Physics, Cosmology and Chaotic Systems," Chaos, Solitons and Fractals, Vol. 7, No. 6, 1996, pp. 877-938. doi:10.1016/0960-0779(96)00002-1

[5] L. Nottale, "Scale Relativity, Fractal Space-Time and Quantum Mechanics," Chaos, Solitons and Fractals, Vol. 4, No. 3, 1994, pp. 361-388. doi:10.1016/0960-0779(94)90051-5

[6] L. Nottale, "Scale Relativity, Fractal Space-Time and Morphogenesis of Structures", Sciences of the Interface, Proceedings of International Symposium in Honor of $O$. Rossler, ZKM Karlsruhe, 2000, p. 38.

[7] L. Nottale, "Scale Relativity," Reprinted from "Scale Invariance and Beyond," In: B. Dubralle, F. Graner and D. Sornette, Eds., Proceedings of Les Houches, EDP Science, 1997, pp. 249-261.

[8] L. Nottale, "Scale Relativity and Quantization of the Universe-I, Theoretical Framework," Astronomy and Astrophysics, Vol. 327, No. 3, 1997, pp. 867-889.

[9] L. Nottale, G. Schumacher and J. Gray, "Scale Relativity and Quantization of the Solar System," Astronomy and Astrophysics, Vol. 322, No. 3, 1997, pp. 1018-1025.

[10] L. Nottale and M. N. Célérier, "Derivation of the Postulates of Quantum Mechanics from the First Principles of Scale 
Relativity," Journal of Physics A: Mathematical and Theoretical, 2007, Vol. 40, No. 48, pp. 14471-14498. doi:10.1088/1751-8113/40/48/012

[11] M.-N. Célérier and L. Nottale, "Electromagnetic KleinGordon and Dirac equations in scale relativity," International Journal of Modern Physics A, Vol. 25, No. 22, 2010, pp. 4239-4253.

[12] L. Nottale, "On the Transition from the Classical to the Quantum Regime in Fractal Space-Time Theory," Chaos, Solitons and Fractals, 2005, Vol. 25, No. 4, pp. 797-803. doi:10.1016/i.chaos.2004.11.071

[13] R. P. Hermann, "Numerical Simulation of a Quantum Particle in a Box," Journal of Physics A: Mathematical and General, Vol. 30, No. 11, 1997, pp. 3967-3975. doi:10.1088/0305-4470/30/11/023

[14] L. I. Schiff., "Quantum Mechanics," 3rd Edition, Int. Student, McGraw-Hill, New York, 1969.

[15] S. Gasiorowicz, "Quantum Physics," John Wiley and Sons, New York, 1974.

[16] J. L. Powell and B. Crasemann, "Quantum Mechanics," Addison-Wesley Co., Inc., Massachusetts, 1961.

[17] C. C. Tannoudji, B. Diue and F. Laloë, "Quantum me- chanics," John Wiley and Sons, New York, 1977.

[18] W. T. Reid, "Riccati Differential Equations," Academic Press, New York, 1972.

[19] F. Charlton, "Integrating Factor for First-Order Differential Equations," Classroom Notes, Aston University, Birmingham, 1998.

[20] N. Bessis and G. Bessis, "Open Perturbation and Riccati Equation: Algebraic Determination of Quartic Anharmonic Oscillator Energies and Eigenfunctions," Journal of Mathematical Physics, Vol. 38, No. 11, 1997, pp. 5483-5492. doi:10.1063/1.532147

[21] G. W. Rogers, "Riccati Equation and Perturbation Expansion in Quantum Mechanics," Journal of Mathematical Physics, Vol. 26, No. 14, 1985, pp. 567-575. doi: $10.1063 / 1.526592$

[22] R. H. Dicke and J. P. Wittke, "Introduction to Quantum Mechanics," Addison-Wesley Co., Inc., Massachusetts, 1960.

[23] D. Počanić, "Bound State in a One-dimensional Square Potential Well in Quantum Mechanics," Classroom Notes, University of Virginia, Charlottesville, 2002. 


\section{Appendix A:}

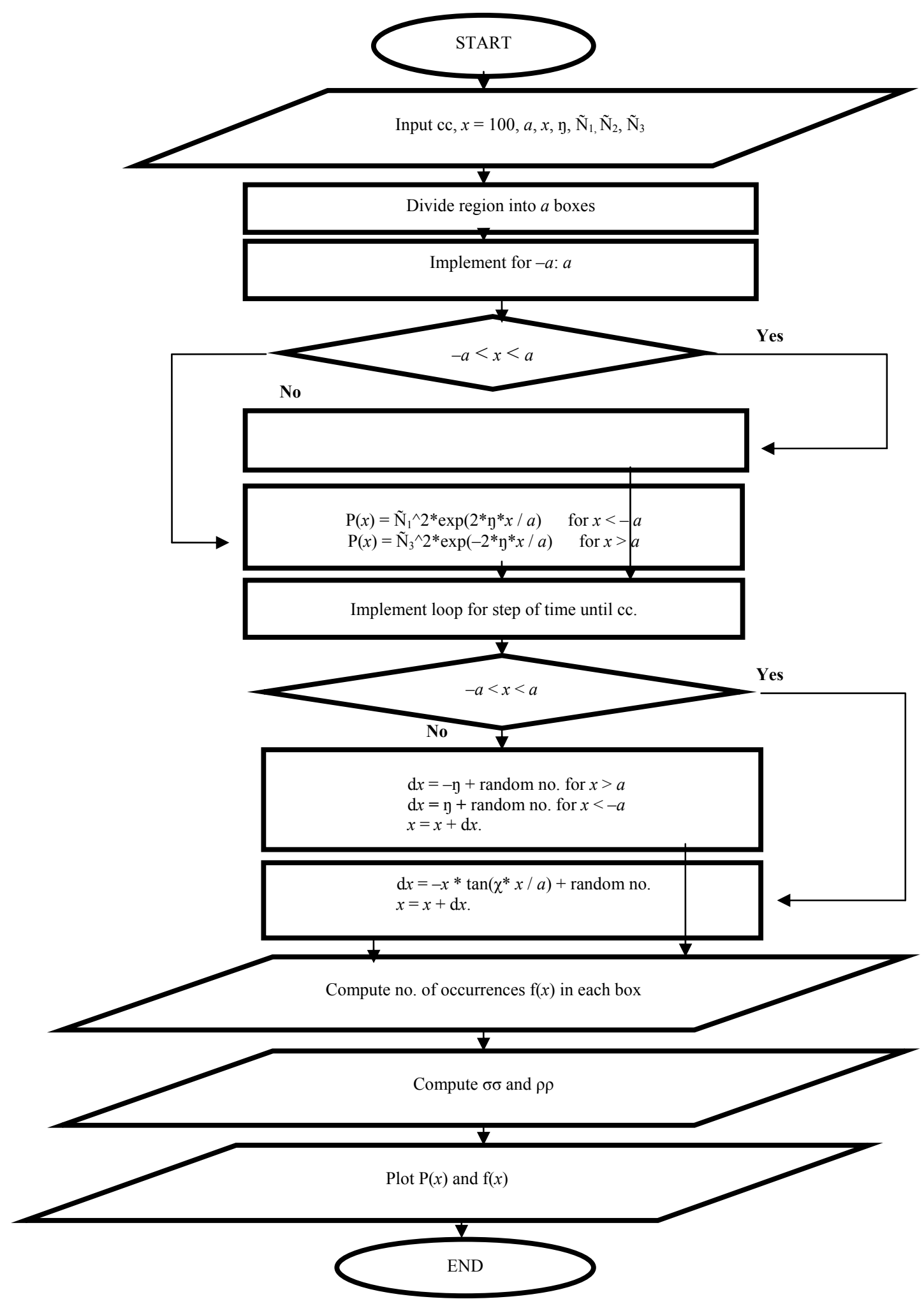

Flowchart 1. A schematic illustration of the different parts of the program that calculates the probability density of a particle in a finite square well potential (even parity). 


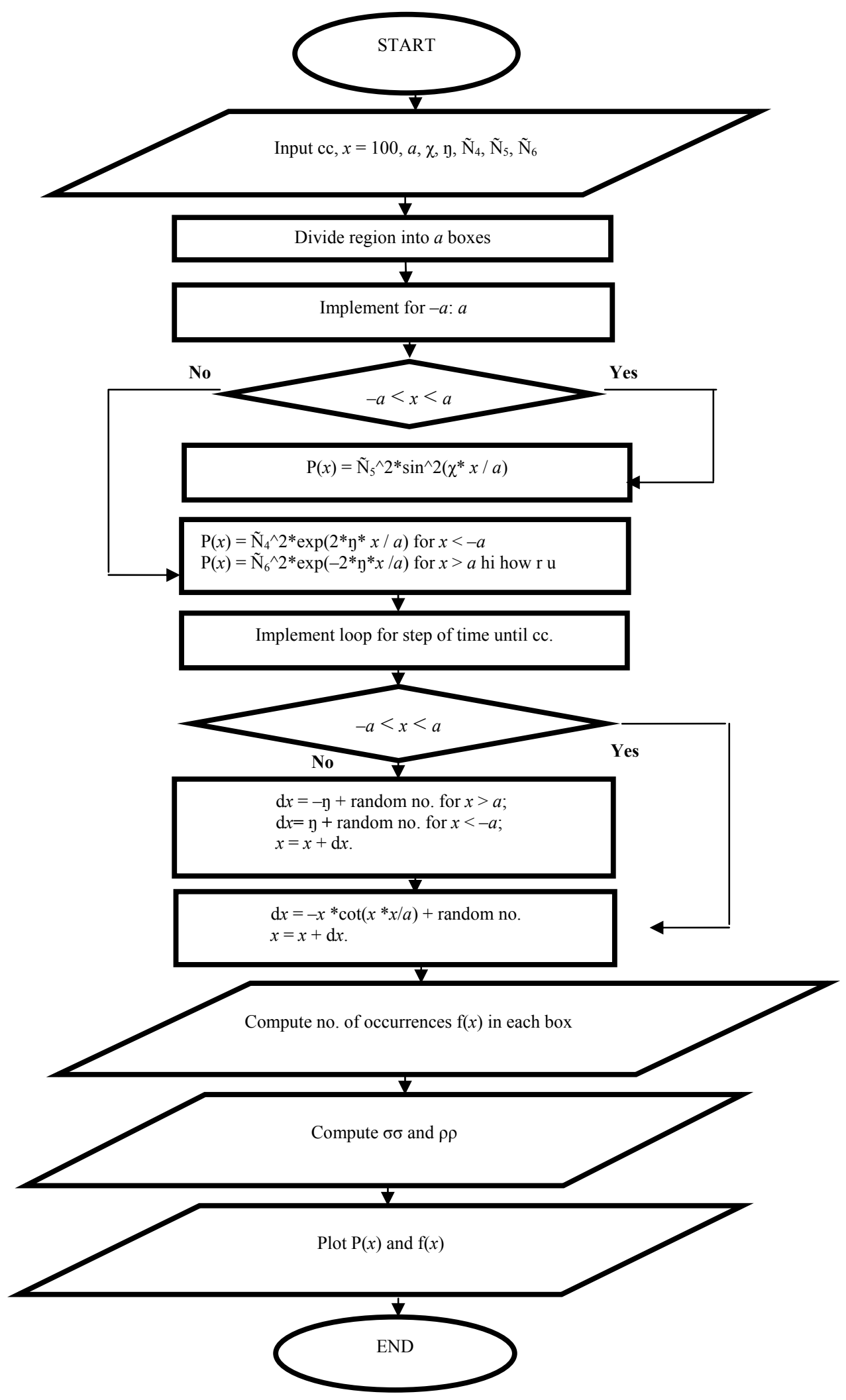

Flowchart 2. A schematic illustration of the different parts of the program that calculates the probability density of a particle in a finite square well potential (odd parity). 